\title{
String Theory at LHC Using Top Quarks From String Balls
}

\author{
Gouranga C. $\operatorname{Nayak}^{1, *}$ \\ 1 Department of Physics, University of Arizona, Tucson, AZ 85721 USA
}

(Dated: June 5, 2018)

\begin{abstract}
According to string theory, string ball is a highly excited long string which decays to standard model particles at the Hagedorn temperature with thermal spectrum. If there are extra dimensions, the string scale can be $\sim \mathrm{TeV}$, and we should produce string balls at CERN LHC. In this paper we study top quark production from string balls at LHC and compare with the parton fusion results at NNLO using pQCD. We find significant top quark production from string balls at LHC which is comparable to standard model pQCD results. We also find that $\frac{d \sigma}{d p_{T}}$ of top quarks from string balls does not decrease significantly with increase in $p_{T}$, whereas it deceases sharply in case of standard model pQCD scenario. Hence, in the absence of black hole production at LHC, an enhancement in top quark cross section and its abnormal $p_{T}$ distribution can be a signature of $\mathrm{TeV}$ scale string physics at LHC. String theory is also studied at LHC via string Regge excitations in the weak coupling limit in model independent framework. Since massive quark production amplitude is not available in string Regge excitations scenario, we compute massless quark production in string Regge excitations scenario and make a clear comparison with that produced from string balls at LHC for a given luminosity.
\end{abstract}

PACS numbers: PACS: 04.70.Bw; 04.70.Dy; 12.38.Bx; 13.85.Ni; 14.65.Ha

*Electronic address: nayak@physics.arizona.edu

Typeset by REVTEX 


\section{INTRODUCTION}

It is now generally accepted that the scale of quantum gravity could be as low as one TeV $[1,12,3,44,5,6,17,8,99,10,11,12,13,14,15,16,17,18$. In the presence of extra dimensions, the string mass scale $M_{s}$ and the Planck mass $M_{P}$ could be around $\sim \mathrm{TeV}$. In this situation we can look forward to search for TeV scale string physics at CERN LHC. One of the most exciting possibility is to search for TeV scale black hole and string ball production at LHC. These 'brane-world' black holes and string balls will be our first window into the extra dimensions of space predicted by string theory, and required by the several brane-world scenarios [19]. There may be many other ways of testing string theory at LHC starting from from brane excitations to various string excitations. The string balls of [20] is just one such model, where the predictions are done in a toy string theory model. In this paper we will focus on studying string theory at LHC based on string balls.

There has been arguments that the black hole stops radiating near Planck scale and forms a black hole remnant [21]. These black hole remnants can be a source of dark matter $[22,23]$. In the absence of a theory of quantum gravity, we can study other scenarios of black hole emissions near the Planck scale. Ultimately, experimental data will determine which scenarios are valid near the Planck scale. In this paper we will study string ball production at LHC in the context of black hole evaporation in string theory. Recently, string theory has given convincing microscopic calculation for black hole evaporation [24, 25].

String theory predicts that a black hole has formed at several times the Planck scale and any thing smaller will dissolve into some thing known as string ball [20]. A string ball is a highly excited long string which emits massless (and massive) particles at Hagedorn temperature with thermal spectrum [26, 27]. For general relativistic description of the back hole to be valid, the black hole mass $M_{B H}$ has to be larger than the Planck mass $M_{P}$. In string theory the string ball mass $M_{S B}$ is larger than the string mass scale $M_{s}$. Typically

$$
\begin{aligned}
& M_{s}<M_{P}<\frac{M_{s}}{g_{s}^{2}} \\
& M_{s}<<M_{S B}<<\frac{M_{s}}{g_{s}^{2}} \\
& \frac{M_{s}}{g_{s}^{2}}<<M_{B H}
\end{aligned}
$$

where $g_{s}$ is the string coupling which can be less than 1 for the string perturbation theory to be valid. Since string ball is lighter than black hole, more string balls are expected to be 
produced at CERN LHC than black holes.

The Hagedorn temperature of a string ball is given by

$$
T_{S B}=\frac{M_{s}}{\sqrt{8} \pi}
$$

where $M_{s} \sim \mathrm{TeV}$ is the string scale. Since this temperature is very high at LHC $(\sim$ hundreds of $\mathrm{GeV})$ we expect more massive particles $\left(M \sim 3 T_{S B}\right)$ to be produced at CERN LHC from string balls.

Top quark is the heaviest observed particle in the standard model. It was discovered in $p \bar{p}$ collisions at $\sqrt{s}=1.8 \mathrm{TeV}$ at Tevatron. Run II of Tevatron at $\sqrt{s}=1.96 \mathrm{TeV}$ has triggered more studies on top quark properties. On the theoretical side, there have been progress on next-to-next leading order (NNLO) pQCD calculations at LHC. Since LHC energy (pp collisions at $\sqrt{s}=14 \mathrm{TeV}$ ) is much larger than the Tevatron energy, LHC is expected to be a top quark factory. This is because gluon-gluon fusion processes at low $x$ are expected to dominate the top quark cross section (about 90 percent).

In this paper we study top quark production at CERN LHC from string balls and make a comparison with the top quark production from the parton fusion processes at NNLO using pQCD. We present the results for the total cross section and $\frac{d \sigma}{d p_{T}}$ of top quarks. There can be significant top quark production from black holes at LHC as well [28]. This is because the black hole temperature increases as its mass decreases whereas the string ball temperature remains constant (see eq. (2) ). On the other hand the string ball mass is smaller than the black hole mass and more string balls are produced at LHC. Hence top quark production at LHC is from two competitive effects: 1) string ball (black hole) production at LHC and 2) top quarks emission from a single string ball (black hole) at LHC. We find that top quark production from string balls can be comparable to that from black holes at LHC. Hence, in the absence of black hole production at LHC, an enhancement in the top quark cross section may be a signature of TeV scale string physics at LHC.

String theory is also studied at LHC via string Regge excitations in the weak coupling limit in model independent framework [36]. Since massive quark production amplitude is not available in string Regge excitations scenario, we compute massless quark production in string Regge excitations scenario and make a clear comparison with that produced from string balls at LHC for a given luminosity.

The paper is organized as follows. In section II we discuss string ball production and its 
decay in string theory and at LHC. In section III we discuss top quark production from string balls at the CERN LHC. Section IV describes top quark production in pQCD at NNLO. In section $\mathrm{V}$ we briefly describe low mass string Regge excitations scenario in partonic collisions at LHC. We present our results and discussions in section VI and conclude in section VII.

\section{STRING BALL PRODUCTION AND DECAY IN STRING THEORY AND AT LHC}

Fundamental scales used in string theory are as follows: $l_{P}$ is the Planck length scale, $l_{s}$ is the quantum length scale of the string, $\alpha^{\prime}=l_{s}^{2}$ is the inverse of the classical string tension, $M_{s}=\frac{1}{l_{s}}$ is the string mass scale and $g_{s}$ is the string coupling. For small string coupling

$$
l_{P} \sim g_{s} l_{s}
$$

In $d=3+n$ space dimensions one obtains

$$
l_{P}^{d-1} \sim g_{s}^{2} l_{s}^{d-1}
$$

According to string theory as black hole shrinks it reaches the correspondence point $[24,25]$

$$
M \leq M_{c} \sim \frac{M_{s}}{g_{s}^{2}}
$$

and makes a transition to a configuration dominated by a highly excited long string. This highly excited long string (known as string ball) continues to lose mass by evaporation at the Hagedorn temperature [26] and "puffs-up" to a larger "random-walk" size which has

observational consequences. Evaporation, still at the Hagedorn temperature, then gradually brings the size of the string ball down towards $l_{s}$.

Production of a highly excited string from the collision of two light string states at high $\sqrt{s}$ can be obtained from the Virasoro-Shapiro four point amplitude by using string perturbation theory. One finds the amplitude

$$
A(s, t)=\frac{2 \pi g_{s}^{2} \Gamma\left[-1-\alpha^{\prime} s / 4\right] \Gamma\left[-1-\alpha^{\prime} t / 4\right] \Gamma\left[-1-\alpha^{\prime} u / 4\right]}{\Gamma\left[2+\alpha^{\prime} s / 4\right] \Gamma\left[2+\alpha^{\prime} t / 4\right] \Gamma\left[2+\alpha^{\prime} u / 4\right]}
$$

with

$$
s+t+u=-16 / \alpha^{\prime}
$$


The production cross section is

$$
\sigma \sim \frac{\pi \operatorname{Res} A\left(\alpha^{\prime} s / 4=N, t=0\right)}{s}=g_{s}^{2} \frac{\pi^{2}}{8} \alpha^{\prime 2} s .
$$

The cross section in eq. (8) saturates the unitarity bounds at around $g_{s}^{2} \alpha^{\prime} s \sim 1$. This implies that the production cross section for string balls grows with $s$ as in eq. (8) only for

$$
M_{s}<<\sqrt{s}<<M_{s} / g_{s}
$$

while for

$$
\sqrt{s}>>M_{s} / g_{s}, \quad \sigma_{S B}=\frac{1}{M_{s}^{2}}
$$

which is constant.

Hence the string ball production cross section in a parton-parton collision is given by [20]

$$
\begin{aligned}
\sigma_{S B} & \sim \frac{g_{s}^{2} M_{S B}^{2}}{M_{s}^{4}}, & M_{s} & <<M_{S B}<<M_{s} / g_{s}, \\
\sigma_{S B} & \sim \frac{1}{M_{s}^{2}}, & M_{s} / g_{s} & <<M_{S B}<<M_{s} / g_{s}^{2} .
\end{aligned}
$$

Highly excited long strings emit massless (and massive) particles at Hagedorn temperature [26]. Hence the conventional description of evaporation in terms of black body radiation can be applied. The emission can take place either in the bulk (in to the closed string) or in the brane (in to open strings). The wavelength

$$
\lambda=\frac{2 \pi}{T_{S B}}
$$

corresponding to Hagedorn temperature is larger than the size of the string ball. So the compact string ball is, to first approximation, a point radiator and, consequently, emits mostly s-waves. This indicates that it decays equally to a particle on the brane and in the bulk. This is because s-wave emission is sensitive only to the radial coordinate and does not make use of the extra angular modes available on the bulk. Since there are many more species of particles $(\sim 60)$ on our brane than our bulk, the string ball decays visibly to standard model particles [20, 29].

However, when string ball puffs-up to a larger random walk size, its spatial extent can approach or exceed the wavelength of the emitted quanta, which implies that it can use 
more of the angular modes that the additional dimensions provide. The average radius of the string ball is

$$
R_{S B} \sim l_{s} \sqrt{M_{S B} l_{s}}
$$

This, however, is a temporary effect: as the string ball decays, its size shrinks towards $l_{s}$, and once again, it becomes a small radiator emitting mostly at brane.

\section{TOP QUARK PRODUCTION FROM STRING BALLS AT LHC}

If string balls are formed at the LHC then they will quickly evaporate by emitting massless (and massive) particles at Hagedorn temperature with thermal spectrum [26, 27]. The emission rate for top quark with mass $M_{t}$, momentum $\vec{p}$ and energy $E=\sqrt{\vec{p}^{2}+M_{t}^{2}}$ from a string ball of temperature $T_{S B}$ is given by

$$
\frac{d N}{d^{3} p d t}=\frac{c_{s} \sigma_{s}}{(2 \pi)^{3}} \frac{1}{\left(e^{\frac{E}{T_{S B}}}+1\right)},
$$

where $\sigma_{s}$ is the area factor [29] and $c_{s}=6$ is the multiplicity factor for top quark.

Note that we do not assume B and L or B-L conservation in this paper. Depending on the assumptions made the probability of the top-quark emission change. In one case the top quark has to be emitted together with an antiquark and in other case it needs to be accompanied by either antiquark or a lepton. In our calculation we do not assume any quantum number conservation. The case of no conservation at all would violate many known bounds on baryon and lepton number conservation. Since we will compare our results with the pQCD NNLO computation at LHC [33, 34] which assumes no quantum number conservation we will assume no quantum number conservation in this paper.

This result in Eq. (14) is for top quark production from a single string ball of temperature $T_{S B}$. To obtain total top quark cross section at LHC we need to multiply the number of top quarks produced from a single string ball with the total string ball production cross section in pp collisions at LHC.

The string ball production cross section in pp collisions at $\sqrt{s}=14 \mathrm{TeV}$ at LHC is given by [3, 15],

$$
\begin{aligned}
\sigma_{S B}^{p p \rightarrow S B+X} & \left(M_{S B}\right)=\sum_{a b} \int_{\tau}^{1} d x_{a} \int_{\tau / x_{a}}^{1} d x_{b} f_{a / p}\left(x_{a}, Q^{2}\right) \\
& \times f_{b / p}\left(x_{b}, Q^{2}\right) \hat{\sigma}^{a b \rightarrow S B}(\hat{s}) \delta\left(x_{a} x_{b}-M_{S B}^{2} / s\right) .
\end{aligned}
$$


In this expression $\hat{\sigma}^{a b \rightarrow S B}(\hat{s})$ is the string ball cross section in partonic collisions which is given by eq. (11), $x_{a}\left(x_{b}\right)$ is the longitudinal momentum fraction of the parton inside the hadron $\mathrm{A}(\mathrm{B})$ and $\tau=M_{S B}^{2} / s$. Energy-momentum conservation implies $\hat{s}=x_{a} x_{b} s=M_{S B}^{2}$. We use $Q=M_{S B}$ as the factorization scale at which the parton distribution functions are measured. $\sum_{a h}$ represents the sum over all partonic contributions where $a, b=q, \bar{q}, g$. We use CTEQ6 [30] PDF to compute the string ball cross section at LHC.

The total top quark production cross section in the process $p p \rightarrow S B+X$ at LHC is then given by

$$
\sigma=N \times \sigma_{S B}
$$

where $\sigma_{\mathrm{SB}}$ is given by eq. (15). To obtain $p_{T}$ distribution we use $d^{3} p=2 \pi d p_{T} p_{T}^{2} d y \cosh y$ in eq. (14) where $y$ is the rapidity.

\section{TOP QUARK PRODUCTION VIA PQCD PROCESSES AT THE LHC}

The top quarks at LHC are mainly produced in $t \bar{t}$ pairs. At the LHC proton-proton collider, the QCD production process involves quark-antiquark and gluon-gluon fusion mechanism. The gluon-gluon fusion processes give the dominant cross section (about 90 percent). This subprocess at high energy is the main reason for larger rate of the cross section compared to Tevatron at Fermilab. The single top quark production occurs via electroweak process. The single top quark production cross section $(\sim 300 \mathrm{pb})$ is smaller compared to $t \bar{t}$ total cross section $(\sim 970 \mathrm{pb})$ at $\mathrm{LHC}$ at $\sqrt{s}=14 \mathrm{TeV}$ pp collisions. Hence we will not consider the single top quark production cross section [31] in this paper. We will consider $t \bar{t}$ pair production using parton fusion processes at LHC and will compare them with the top quark production cross section from string balls.

At the next-to-next-to-leading order (NNLO) one needs to compute the following partonic subprocesses. On the leading-order (LO) level we have

$$
q+\bar{q} \rightarrow t \bar{t}, \quad g+g \rightarrow t \bar{t}
$$

In NLO we have in addition to the one-loop virtual corrections to the above reaction the following two-to-three body processes

$$
q+\bar{q} \rightarrow t \bar{t}+g, \quad g+q(\bar{q}) \rightarrow t \bar{t}+q(\bar{q}), \quad g+g \rightarrow t \bar{t}+g .
$$


At NNLO level we receive the two-loop virtual corrections to the LO processes in eq. (17) and one-loop virtual corrections to NLO reactions in eq. (18). To these contribution one has to add the results obtained from the following two-to-four body reactions

$$
\begin{array}{lc}
g+g \rightarrow t \bar{t}+g+g, & g+g \rightarrow t \bar{t}+q+\bar{q}, \\
g+q(\bar{q}) \rightarrow t \bar{t}+q(\bar{q})+g, & \\
q+\bar{q} \rightarrow t \bar{t}+g+g, & q+\bar{q} \rightarrow t \bar{t}+q+\bar{q}, \\
q+q \rightarrow t \bar{t}+q+q, & \bar{q}+\bar{q} \rightarrow t \bar{t}+\bar{q}+\bar{q}, \\
q_{1}+q_{2} \rightarrow t \bar{t}+q_{1}+q_{2}, & q_{1}+\bar{q}_{2} \rightarrow t \bar{t}+q_{1}+\bar{q}_{2} .
\end{array}
$$

After the phase space integrals has been done the partonic cross section $\hat{\sigma}$ is rendered finite by coupling constant renormalization, operator renormalization and the removal of collinear divergences. The renormalization scale $\mu_{R}$ is set equal to the mass factorization scale $\mu_{F}$. The cross section for top quark production in proton-proton collisions at the LHC is given by

$$
d \sigma=\sum_{a, b=q, \bar{q}, g} \int d x_{1} \int d x_{2} f_{a}\left(x_{1}, \mu_{F}^{2}\right) f_{b}\left(x_{2}, \mu_{F}^{2}\right) d \hat{\sigma}_{a b}
$$

where $d \hat{\sigma}_{a b}$ is the partonic level differential cross section for top quark production. For the details, see [32, 33, 34]. Reviews of present status of top quark physics at LHC can be found in [35].

\section{PARTON PRODUCTION IN $2 \rightarrow 2$ PROCESSES VIA STRING REGGE EX- CITATIONS}

If the string mass scale $M_{s} \sim \mathrm{TeV}$, we can also expect to discover string Regge excitations with masses of order $M_{s}$ in $2 \rightarrow 2$ partonic processes at LHC in the weak coupling limit in a model independent framework [36]. In this case a whole tower of infinite string excitations will open up and the new particles follow the well known Regge trajectories of vibrating string

$$
j=j_{0}+\alpha^{\prime} M^{2}
$$

with spin $j$. These stringy states will lead to new contributions to standard model scattering processes. This is based on the extensions of standard model where open strings ends on 
D-branes, with gauge bosons due to strings attached to stacks of D-branes and chiral matter due to strings stretching between intersecting D-branes [37].

Dijet production in the string resonance scenario in partonic collisions at LHC is studied in [36]. The $2 \rightarrow 2$ partonic scattering amplitudes are computed at the leading order in string perturbation theory [38]. In this calculation $\hat{s}+\hat{t}+\hat{u}=0$ is used which is the case for massless partons in the initial and final states in the $2 \rightarrow 2$ partonic scattering processes. Here $\hat{s}, \hat{t}$ and $\hat{u}$ are the Mandelstam variables at partonic level. For top quark production one needs to extend this string Regge formalism to the case $\hat{s}+\hat{t}+\hat{u} \neq 0$ to calculate the amplitude. Since massive quark production amplitude is not available in string Regge excitations scenario, we will compute massless quark production in string Regge excitations scenario and will make a clear comparison with that produced from string balls at LHC for a given luminosity. It can be mentioned that a similar situation exists in AdS/CFT scenario as well, where gluon scattering amplitude [39] and massless quark scattering amplitudes are studied [40]. The partonic scattering amplitude for the massive quark production in the final state in the AdS/CFT scenario is expected to be complicated and has not been studied so far.

Since the gluon fusion process is dominant at LHC we will consider the process $g g \rightarrow q \bar{q}$ via string Regge excitation in this paper. The matrix element square for this process is given by [36]

$$
\begin{aligned}
& |M(g g \rightarrow q \bar{q})|^{2}=\frac{7}{24} \frac{16 \pi^{2} \alpha_{s}^{2}}{M_{s}^{4}} N_{f} \times \\
& {\left[W_{g^{*}}^{g g \rightarrow q \bar{q}} \frac{\hat{u} \hat{t}\left(\hat{u}^{2}+\hat{t}^{2}\right)}{\left(\hat{s}-M_{s}^{2}\right)^{2}+\left(\Gamma_{g^{*}}^{J=2} M_{s}\right)^{2}}+W_{C^{*}}^{g g \rightarrow q \bar{q}} \frac{\hat{u} \hat{t}\left(\hat{u}^{2}+t^{2}\right)}{\left(\hat{s}-M_{s}^{2}\right)^{2}+\left(\Gamma_{C^{*}}^{J=2} M_{s}\right)^{2}}\right]}
\end{aligned}
$$

where $\alpha_{s}$ is the QCD coupling constant and

$$
\begin{array}{ll}
W_{g^{*}}^{g g \rightarrow q \bar{q}}=0.24, & W_{C^{*}}^{g g \rightarrow q \bar{q}}=0.76, \\
\Gamma_{g^{*}}^{J=2}=45\left(M_{s} / \mathrm{TeV}\right) \mathrm{GeV}, & \Gamma_{C^{*}}^{J=2}=75\left(M_{s} / \mathrm{TeV}\right) \mathrm{GeV} .
\end{array}
$$

The differential cross section for jet production in pp collisions at LHC is given by

$$
E \frac{d \sigma}{d^{3} p}=\int d x_{1} \int d x_{2} f\left(x_{1}, Q^{2}\right) f\left(x_{2}, Q^{2}\right) \frac{\hat{s}}{\pi} \frac{d \hat{\sigma}}{d \hat{t}} \delta(\hat{s}+\hat{t}+\hat{u})
$$

where $\frac{d \hat{\sigma}}{d \hat{t}}$ is the partonic level differential cross section. This gives for the quark jet production

$$
\frac{d \sigma}{d p_{T}}=\frac{p_{T}}{8 \pi s} \int d y \int d y_{2} \frac{1}{\hat{s}} f_{g}\left(x_{1}, Q^{2}\right) f_{g}\left(x_{2}, Q^{2}\right) \times|M(g g \rightarrow q \bar{q})|^{2}
$$


where

$$
x_{1}=\frac{p_{T}}{\sqrt{s}}\left[e^{y}+e^{y_{2}}\right], \quad x_{2}=\frac{p_{T}}{\sqrt{s}}\left[e^{-y}+e^{-y_{2}}\right] .
$$

$|M(g g \rightarrow q \bar{q})|^{2}$ is given by eq. (22) for the process $g g \rightarrow q \bar{q}$ in the string Regge excitation

scenario [36]. We will compare eq. (25) with $\frac{d \sigma}{d p_{T}}$ from eq. (16) for massless quark production from string balls at LHC. We have used CTEQ6 PDF [30] in our calculation.

\section{RESULTS AND DISCUSSIONS}

In this section we will compute the top quark production cross section from string balls at $\sqrt{s}=14 \mathrm{TeV}$ in pp collisions and will compare them with the top quark production via parton fusion processes at NNLO. The top quark production from string ball is described in section III. For the string ball production we choose the factorization and normalization scale to be the mass of the string ball. As the temperature of the string ball is very large there is not much difference in the top quark production cross section from string balls if the top quark mass $M_{t}$ is increased from 165 to $180 \mathrm{GeV}$. String ball mass $M_{S B}$ should be larger than the string scale $M_{S}$. We take

$$
M_{S B} \geq 3 M_{s}, \quad g_{s}=0.3
$$

in our calculation [27].

In Fig. 1 we present the string ball production cross section at the LHC. The $y$-axis is the string ball production cross section $\sigma_{S B}$ in $\mathrm{pb}$ and the $x$-axis is the string ball mass $M_{S B}$ in $\mathrm{TeV}$. The upper curve is for string scale $M_{s}=1 \mathrm{TeV}$. The solid line in the upper curve is for the string ball mass in the range $M_{s}<<M_{S B}<<M_{s} / g_{s}$ and the dashed line is for $M_{s} / g_{s}<<M_{S B}<<M_{s} / g_{s}^{2}$, see eq. (11). The middle curve is for string scale $M_{s}=2 \mathrm{TeV}$. The dot-dashed line in the middle curve is for the string ball mass in the range $M_{s}<<$ $M_{S B}<<M_{s} / g_{s}$ and the solid line in the middle curve is for $M_{s} / g_{s}<<M_{S B}<<M_{s} / g_{s}^{2}$. The lower curve is for string scale $M_{s}=3 \mathrm{TeV}$. The dotted line in the lower curve is for the string ball mass in the range $M_{s}<<M_{S B}<<M_{s} / g_{s}$ and the dot-dashed-dashed line in the lower curve is for $M_{s} / g_{s}<<M_{S B}<<M_{s} / g_{s}^{2}$. As can be seen from the figure the cross sections decrease rapidly when both the string mass scale $M_{s}$ and string ball mass $M_{S B}$ increases. These string ball production cross sections will be multiplied with the number 


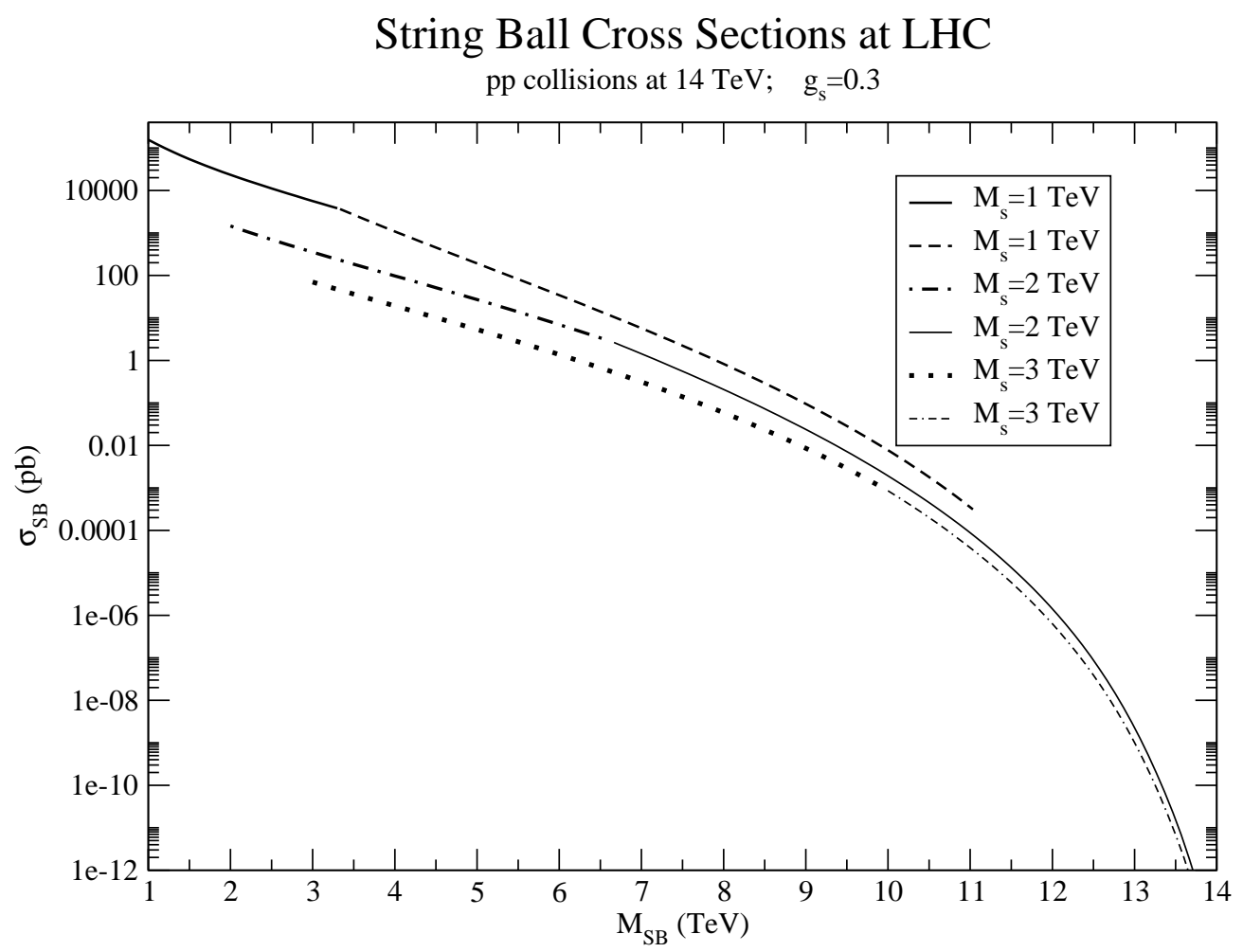

FIG. 1: Total cross sections for string ball production at the LHC.

of top quarks produced from a single string ball to obtain the top quark production cross section from string balls at the CERN LHC.

In Fig. 2 we present results for the average number of top quarks produced from a single string ball as a function of top quark mass. The $y$-axis is the average number of top quark production from a single string ball and the $x$-axis is the mass of the top quark in $\mathrm{GeV}$. The solid, dashed and dotted lines are for string ball masses equal to 10, 6 and $3 \mathrm{TeV}$ respectively. Unlike black hole case, the average number of top quarks produced from a string ball is larger for larger mass string balls. This is because the Hagedorn temperature, eq. (2), is independent of the string ball mass whereas the black hole temperature decreases as black hole mass increases. The increase in number of top quarks is due to the increase in area of the string ball, see eqs. (13) and (14). This is the case for emission from a single string ball. The string ball production cross section itself decreases at LHC as the mass of the string ball increases, see Fig.1. Hence the total cross section of top quark production from string balls at LHC is a competitive effect from the above two factors (see eq. (16)) .

In Fig. 3 we present total top quark production cross section from string balls and compare 


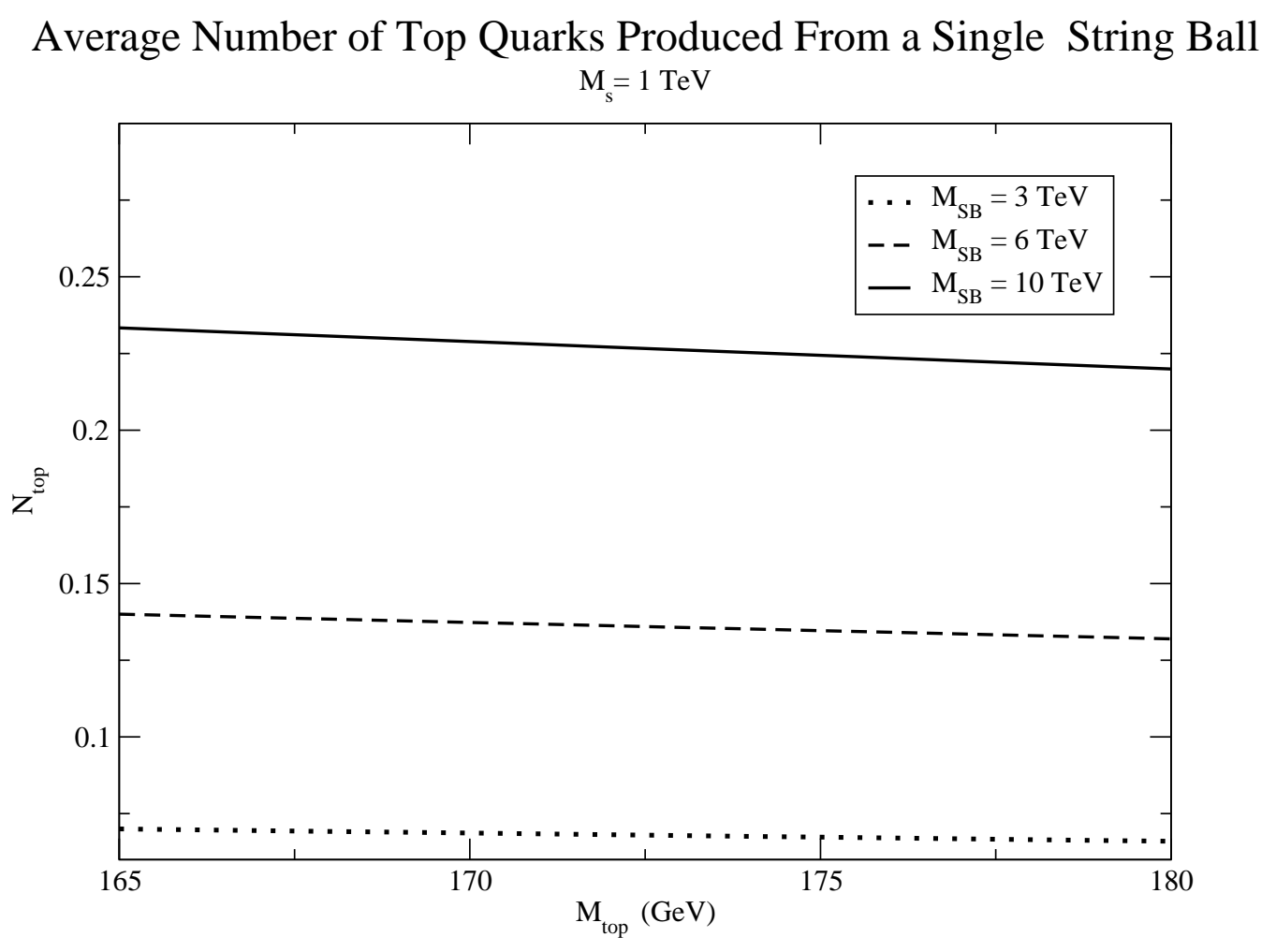

FIG. 2: Average Number of top quark production from a single string ball at LHC.

them with the pQCD predictions at NNLO. The former is given for three different choices of the string ball mass, namely $M_{S B}=3,6$ and $10 \mathrm{TeV}$ respectively. We plot for comparison the NNLO top quark cross section with $\mu_{F}=\mu_{R}=M_{t}[33]$. The dotted line for NNLO curve is for MRST $2006 \mathrm{PDF}$ and the thin-solid line for NNLO curve is for CTEQ6.6 PDF. The thick-solid line is for string ball mass equal to $3 \mathrm{TeV}$, the dashed line is for string ball mass equal to $6 \mathrm{TeV}$ and the dot-dashed line is for string ball mass equal to $10 \mathrm{TeV}$. For larger value of string mass scale $M_{s}$ the cross section becomes even smaller and hence we do not plot them. It is clear that the total top quark cross section from string balls is comparable to $\mathrm{pQCD}$ cross section for small value of string mass scale $\left(M_{s} \sim 1 \mathrm{TeV}\right)$ and string ball mass $\left(M_{S B} \sim 3 \mathrm{TeV}\right)$ and is not sensitive to the increase in top quark mass $M_{t}$.

In Fig.4 we present $\frac{d \sigma}{d p_{T}}$ of top quark production from string balls at LHC and compare them with the pQCD predictions at NNLO. The top quark mass is chosen to be $175 \mathrm{GeV}$. The $\frac{d \sigma}{d p_{T}}$ of top quark from string balls is given for three different choices of the string ball mass, namely $M_{S B}=3,6$ and $10 \mathrm{TeV}$ respectively with string mass scale $M_{s}=1 \mathrm{TeV}$ in each case. We plot for comparison the NNLO results for $\frac{d \sigma}{d p_{T}}$ of top quark using pQCD [34]. 


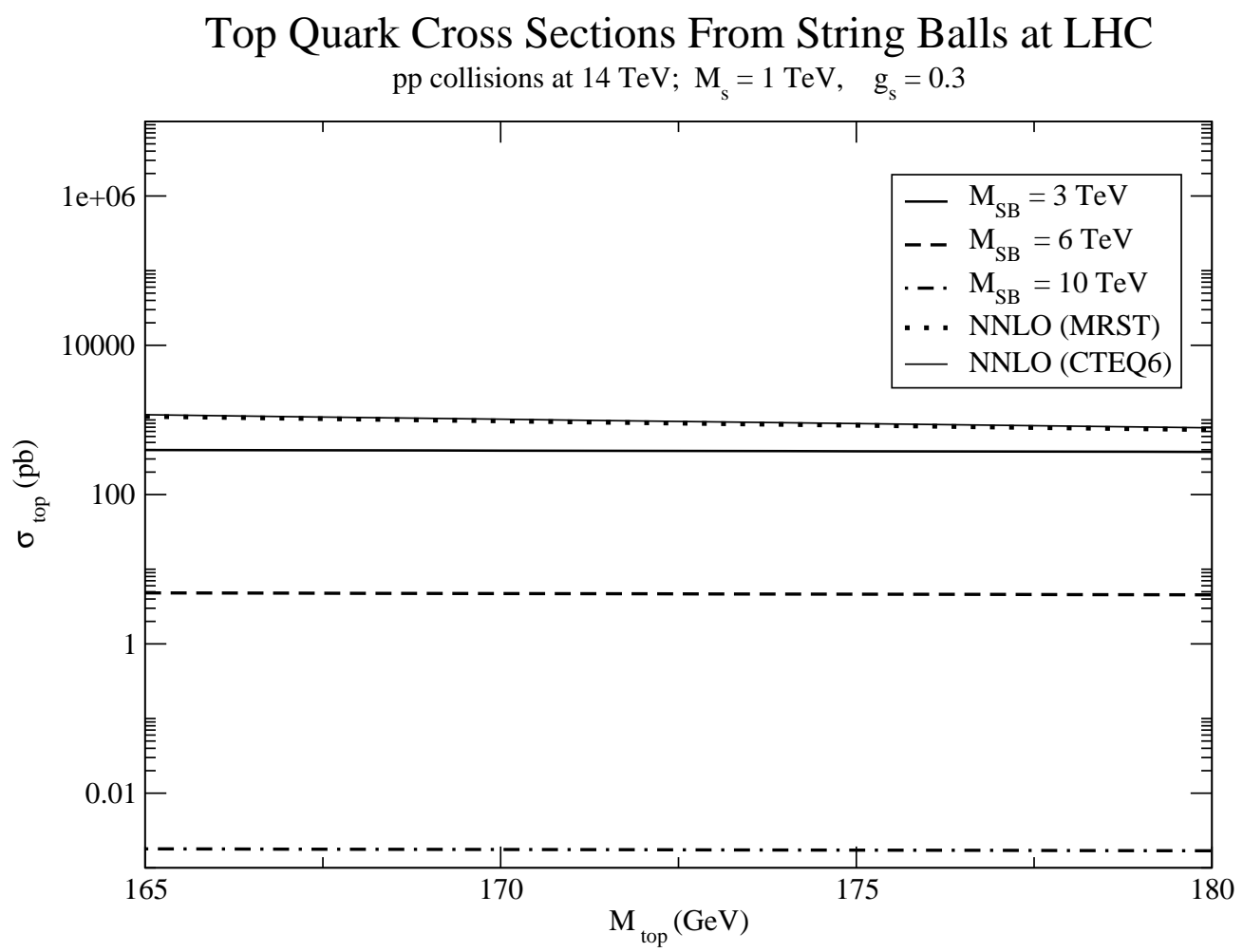

FIG. 3: Total cross section for top quark production at LHC from string balls and from direct pQCD processes at NNLO.

The dashed line is the NNLO pQCD result. The solid line is for string ball mass equal to $3 \mathrm{TeV}$, the dotted line is for string ball mass equal to $6 \mathrm{TeV}$ and the dot-dashed line is for string ball mass equal to $10 \mathrm{TeV}$. For larger value of string scale $M_{s}$ the cross section becomes even smaller and hence we do not plot them. It is clear that the $\frac{d \sigma}{d p_{T}}$ of the top quark via string ball production is larger than the standard model pQCD predictions for larger values of $p_{T}\left(p_{T}>250 \mathrm{GeV}\right)$ of top quark and for smaller value of string ball mass $\left(M_{S B} \sim 3 \mathrm{TeV}\right)$. For larger values of $p_{T}$, the $\frac{d \sigma}{d p_{T}}$ of top quark from string balls does not sharply decrease with increasing $p_{T}$, whereas in case of NNLO pQCD processes it decreases sharply. This fact can be used to distinguish between top quark production from string balls and from parton fusion processes at NNLO in PQCD at LHC.

Since massive quark production amplitude is not available in string Regge excitations scenario, in Fig. 5 we present $\frac{d \sigma}{d p_{T}}$ of massless quark production from string balls and compare with the process $g g \rightarrow q \bar{q}$ at LHC in the string Regge excitation scenario [36] by using eq. (25). We have considered single quark flavor which corresponds to the massless 


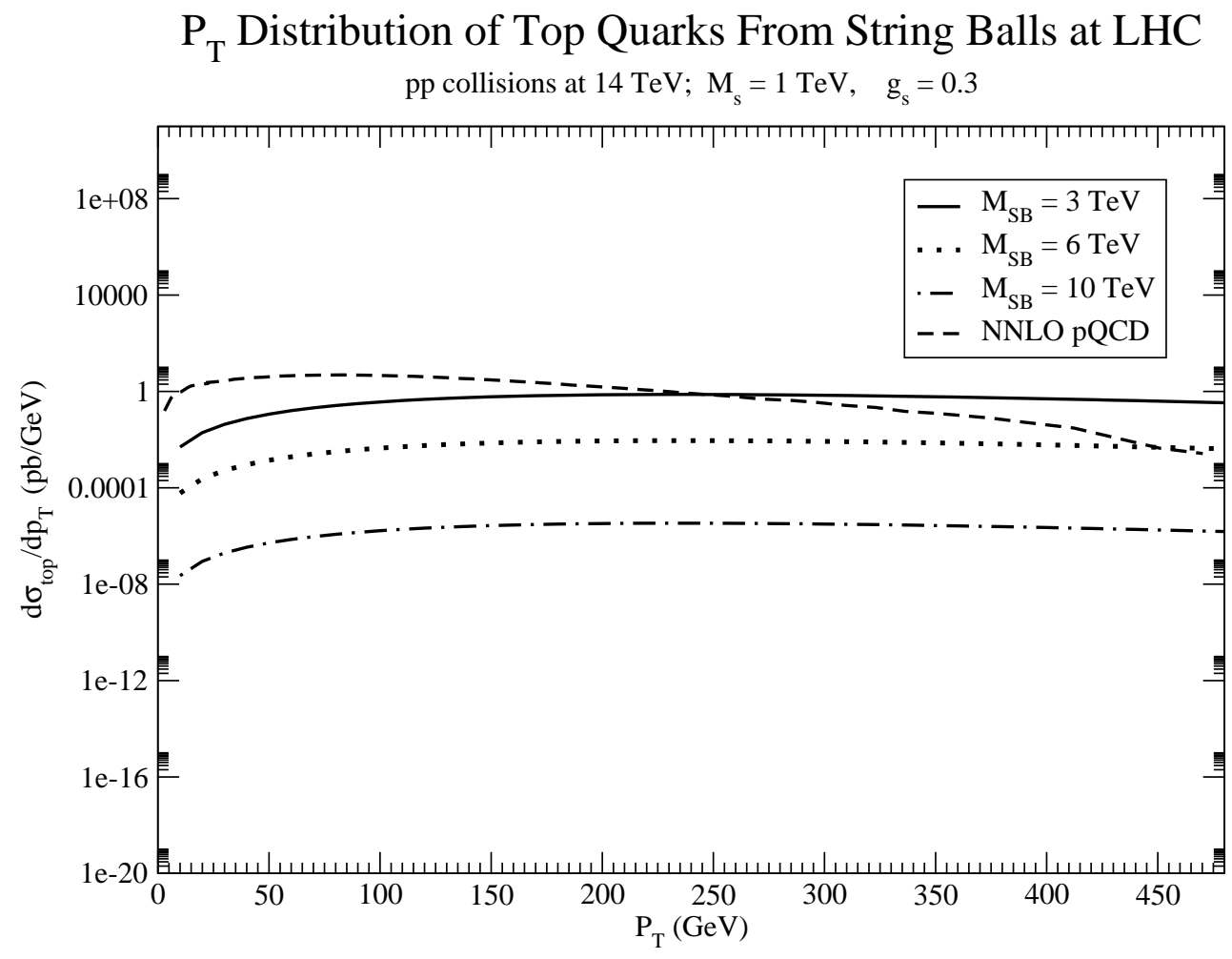

FIG. 4: Transverse momentum distribution of top quark production at LHC from string balls and from direct $\mathrm{pQCD}$ processes at NNLO.

limit of the top quark. See section $\mathrm{V}$ for details. The thick solid line is for string mass scale $M_{s}=1 \mathrm{TeV}$ and string ball mass $M_{S B}=3 \mathrm{TeV}$. The dotted line is for $M_{s}=1 \mathrm{TeV}$ and $M_{S B}=6 \mathrm{TeV}$. The dot-dashed line is for $M_{s}=1 \mathrm{TeV}$ and $M_{S B}=10 \mathrm{TeV}$. For comparison we present $\frac{d \sigma}{d p_{T}}$ of massless quark production in the string Regge excitation scenario. The thin solid, dashed and dot-dashed-dashed lines are in the string Regge excitation scenario for $M_{s}=1,2$ and $4 \mathrm{TeV}$ respectively. It can be seen that the resonances are observed in case of string excitation scenario which is absent in the string ball scenario.

In Fig. 6 we present number of quark jets per $\mathrm{GeV}$ from string balls at LHC and compare with the process $g g \rightarrow q \bar{q}$ at LHC in the string Regge excitation scenario [36] with the luminosity of $10 \mathrm{pb}^{-1}$. The thick solid line is for string mass scale $M_{s}=1 \mathrm{TeV}$ and string ball mass $M_{S B}=3 \mathrm{TeV}$. The dotted line is for $M_{s}=1 \mathrm{TeV}$ and $M_{S B}=6 \mathrm{TeV}$. The dot-dashed line is for $M_{s}=1 \mathrm{TeV}$ and $M_{S B}=10 \mathrm{TeV}$. For comparison we present number of quark jets per $\mathrm{GeV}$ in the string Regge excitation scenario. The dot-dashed-dashed and dot-dot-dashed lines are in the string Regge excitation scenario for $M_{s}=1$ and $2 \mathrm{TeV}$ respectively. We also 


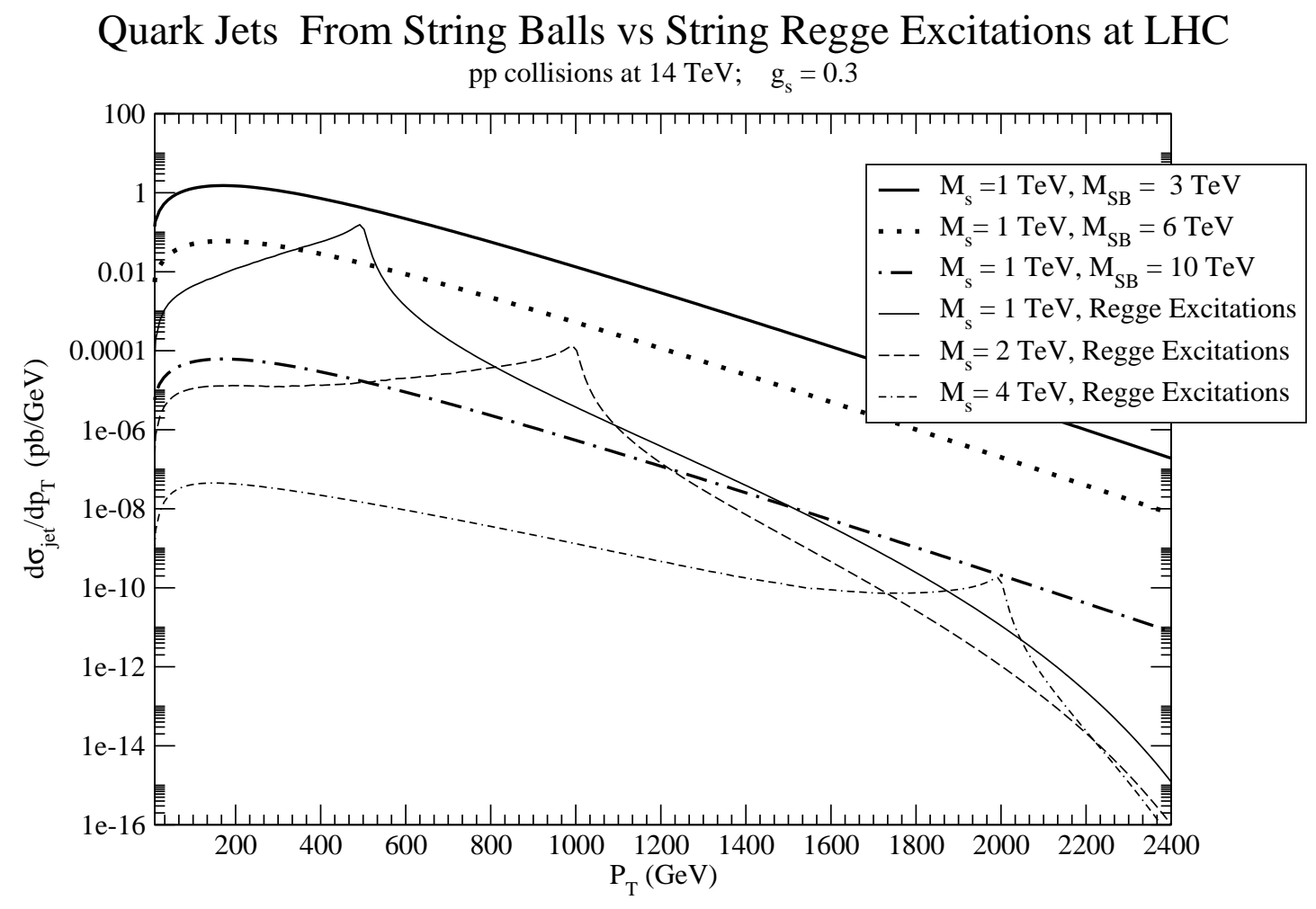

FIG. 5: Transverse momentum distribution of quark jet production from string balls and from string Regge excitations at LHC.

present the results of the QCD jets at the CMS detector at LHC. The thin solid line is the result for CMS QCD jets taken from [41] with the same luminosity of $10 \mathrm{pb}^{-1}$. It can be seen that the resonances are observed in case of string excitation scenario which is absent in the string ball scenario and in QCD jets scenario.

\section{CONCLUSIONS}

In summary, we have computed top quark production cross section and its $p_{T}$ distribution from string balls in proton-proton collisions at the LHC at $\sqrt{s}=14 \mathrm{TeV}$ in the context of string theory and $\mathrm{TeV}$ scale gravity. We have compared the result with the pQCD cross sections at NNLO. As the temperature of the string ball is large there is a huge amount of top quark production from string balls at the LHC if the string scale is $\sim 1 \mathrm{TeV}$ and the string ball mass is $\sim 3 \mathrm{TeV}$. We have found that, unlike standard model predictions, the top quark production cross section from string ball is not sensitive to the increase in top quark mass. We have also found that $\frac{d \sigma}{d p_{T}}$ of top quark from string balls does not sharply decrease 


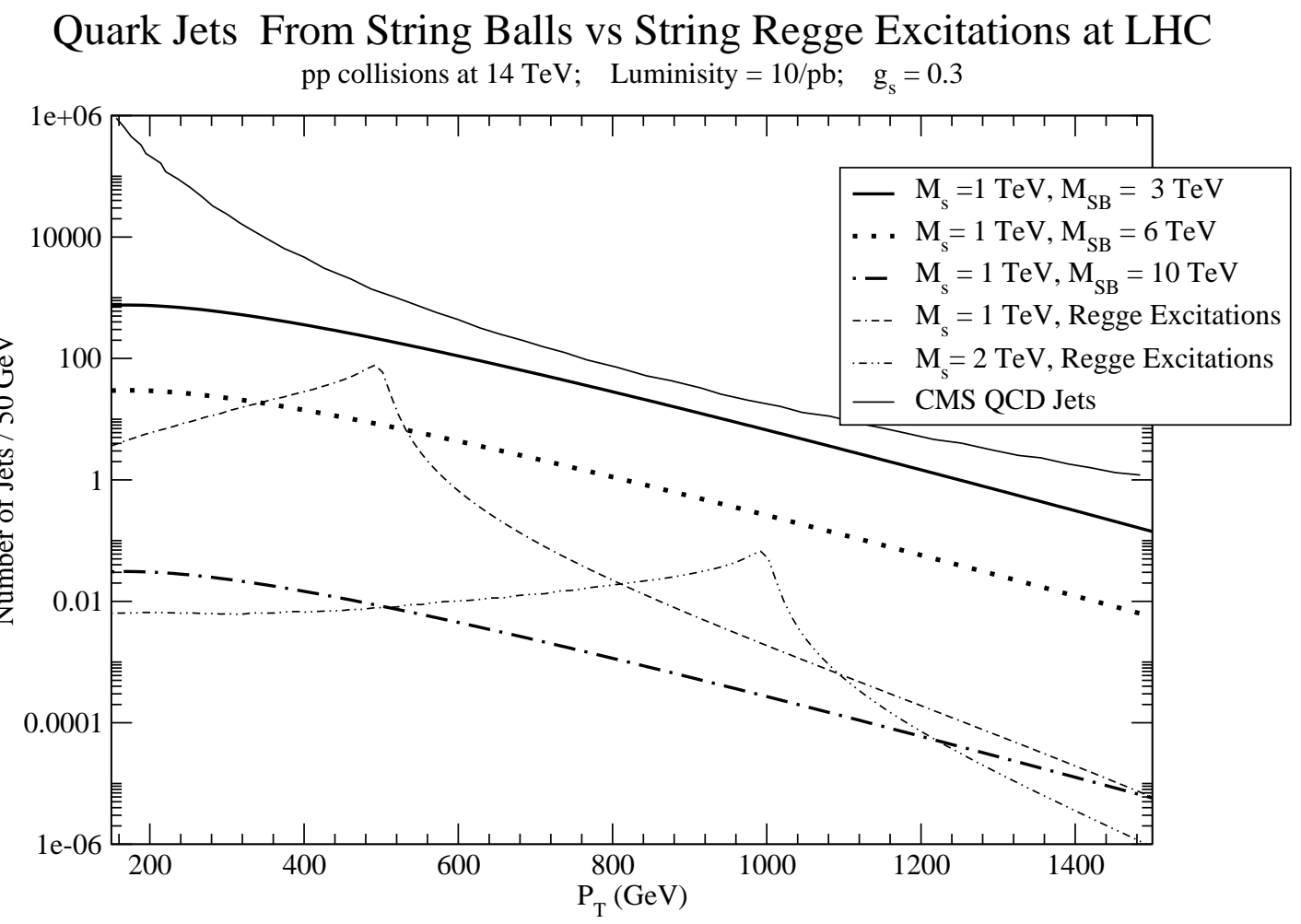

FIG. 6: Number of quark jets per $\mathrm{GeV}$ (in $50 \mathrm{GeV} p_{T}$ bin) as a function of $p_{T}$ from string balls and from string Regge excitations at LHC. The luminosity used is $10 \mathrm{pb}^{-1}$. CMS QCD jets are from [41].

with increasing $p_{T}$, whereas in standard model processes it decreases sharply. Hence, in the absence of black hole production at LHC, an enhancement in top quark cross section and its abnormal $p_{T}$ distribution can be a signature of TeV scale string physics at LHC.

String theory is also studied at LHC via string Regge excitations in the weak coupling limit in model independent framework [36]. Since massive quark production amplitude is not available in string Regge excitations scenario, we have computed massless quark production in string Regge excitations scenario and have made a clear comparison with that produced from string balls at LHC for a given luminosity.

String balls might also be produced in $\mathrm{PbPb}$ collisions with a larger rate [15]. In this case there can be additional effects of quark-gluon plasma [42] on the string ball radiation [26]. 


\section{Acknowledgments}

This work was supported in part by Department of Energy under contracts DE-FG0291ER40664, DE-FG02-04ER41319 and DE-FG02-04ER41298.

[1] N. Arkani-Hamed, S. Dimopoulos and G.R. Dvali, Phys. Lett. B429, 263 (1998); Phys. Rev. D59,086004 (1999); I. Antoniadis, N. Arkani-Hamed, S. Dimopoulos and G.R. Dvali, Phys. Lett. B436, 257 (1998); L. Randall and R. Sundrum, Phys. Rev. Lett. 83, 3370 (1999); 83, 4690 (1999).

[2] T. Banks and W. Fischler, hep-th/9906038.

[3] S. Dimopoulos and G. Landsberg, Phys. Rev. Lett. 87, 161602 (2001); P.C. Argyres, S. Dimopoulos and J. March-Russell, Phys. Lett. B441, 96 (1998).

[4] S. B. Giddings and S. Thomas, Phys. Rev. D 65, 056010 (2002).

[5] S. B. Giddings, in Proc. of the APS/DPF/DPB Summer Study on the Future of Particle Physics (Snowmass 2001) ed. R. Davidson and C. Quigg, hep-ph/0110127; M.B. Voloshin, Phys. Lett. B518, 137 (2001); Phys. Lett. B524, 376 (2002).

[6] D. M. Eardley and S. B. Giddings, Phys. Rev. D 66, 044011 (2002); S.N. Solodukhin, Phys. Lett. B533, 153 (2002); A. Jevicki and J. Thaler, Phys. Rev. D66, 024041 (2002).

[7] L. Anchordoqui and H. Goldberg, Phys. Rev. D 65, 047502 (2002); Phys. Rev. D67, 064010 (2003).

[8] R. Casadio and B. Harms, Int. J. Mod. Phys. A 17, 4635 (2002).

[9] K. Cheung, Phys. Rev. D 66, 036007 (2002); Phys. Rev. Lett. 88, 221602 (2002); K. Cheung and Chung-Hsien Chou, Phys. Rev. D 66, 036008 (2002).

[10] Y. Uehara, Mod. Phys. Lett. A 17, 1551 (2002).

[11] Seong Chan Park and H.S. Song, J. Korean Phys. Soc. 43, 30 (2003).

[12] M. Bleicher, S. Hofmann, S. Hossenfelder and H. Stoecker, Phys. Lett. B 548, 73 (2002); S. Hossenfelder, S. Hofmann, M. Bleicher and H. Stoecker, Phys. Rev. D 66, 101502 (2002).

[13] I. Mocioiu, Y. Nara and I. Sarcevic, Phys. Lett. B557, 87 (2003); V. Frolov and D. Stojkovic, Phys. Rev. D 68, 064011 (2003); 67, 084004 (2003); 66, 084002 (2002); D. Ida and S. C. Park, Phys. Rev. D 67, 064024 (2003); 69, 049901 (E) (2004); B. Kol, hep-ph/0207037; M. 
Cavaglia, S. Das and R. Maartens, Class. Quant. Grav. 20, 1205 (2003); M. Cavaglia and S. Das, Class. Quant. Grav. 21, 4511 (2004); S. Hosenfelder, Phys. Lett. B598, 92 (2004); A. Ringwald, Fortsch. Phys. 51, 830 (2003).

[14] T. Han, G. D. Kribs and B. McElrath, Phys. Rev. Lett. 90, 031601 (2003); P. Kanti, Int. J. Mod. Ohys. A 19, 4899 (2004); G. Landsberg, [arXiv; hep-ph/0211013]; M. Cavaglia, Int. J. Mod. Phys. A18, 1843 (2003); and H. Stoecker, AIP Conf.Proc.947:376-382,2007; L. A. Anchordoqui, H. Goldberg and S. Nawata, arXiv:0804.2013 [hep-ph]; D-C. Dai, G. Starkman, D. Stojkovic, C. Issever, E. Rizvi and J. Tseng, Phys.Rev.D77:076007,2008.

[15] A. Chamblin and G. C. Nayak, Phys. Rev. D 66, 091901 (2002).

[16] L. A. Anchordoqui, J. L. Feng, H. Goldberg and A. D. Shapere, Phys.Rev. D65 (2002) 124027; Phys. Rev. D 66, 103002 (2002); J. L. Feng and A. D. Shapere, Phys. Rev. Lett. 88, 021303 (2002); L. A. Anchordoqui, T. Paul, S. Reucroft and J. Swain, Int. J. Mod. Phys. A 18, 2229 (2003); A. Chamblin, F. Cooper and G. C. Nayak, Phys. Rev. D 70, 075018 (2004); G. C. Nayak and J. Smith, Phys. Rev. D 74, 014007 (2006), Haunting Higgs Boson in Mini Black Holes, issue 2562, New Scientist, 28 July 2006, p12; L. Anchordoqui and H. Goldberg, Phys. Rev. D 67, 064010 (2003); G. C. Nayak, hep-ph/0211395. A. Chamblin, F. Cooper and G. C. Nayak, Phys. Rev. D 69, 065010 (2004).

[17] R. Emparan, M. Masip and R. Rattazzi, Phys. Rev. D 65, 064023 (2002).

[18] A. Ringwald and H. Tu, Phys. Lett. B 525, 135 (2002).

[19] A. Chamblin, S. W. Hawking and H. S. Reall, Phys. Rev. D 61, 065007 (2000); R. Emparan, G. T. Horowitz and R. C. Myers, J. High Energy Phys. 01, 07 (2000).

[20] S. Dimopoulos and R. Emparan, Phys. Lett. B526 (2002) 393, hep-ph/0108060.

[21] M. Cavaglia and S. Das, Class. Quant. Grav. 21 (2004) 4511; M. Cavaglia, S. Das and R. Maartens, Class. Quant. Grav. 20 (2003) L205.

[22] D. Baumann, P. Steinhardt and N. Turok, hep-th/0703250, S. Alexander and P. Meszaros, hep-th/0703070; P. Chen, New Astronomy Reviews 49 (2005) 233; Nucl. Phys. B (Proc. Suppl.) 124 (2003) 103.

[23] G. C. Nayak, arXiv:0901.3358 [hep-ph].

[24] L. Susskind, hep-th/9309145.

[25] M. J. Bowick, L. Smolin and L. C. Wijewardhana, Phys. Rev. Lett. 56 (1986) 424; E. Halyo, B. Kol, A. Rajaraman and L. Susskind, Phys. Lett. B 401 (1997) 15; G. Veneziano, in NATO 
Advanced Study Workshop on Hot Hadronic Matter: Theory and Experiment, Divonne-lesBains, France, 27 Jun-1 jul 1994; G. T. Horowitz and J. Polchinski, Phys. Rev. D55 (1997) 6189; Phys. Rev. D57 (1998) 2557; T. Damour G. Veneziano, Nucl. Phys. B568 (2000) 93.

[26] D. Amati and J. G. Russo, Phys. Lett. B454 (1999) 207, hep-th/9901092.

[27] D. M. Gingrich and K. Martell, Phys. Rev. D78 (2008) 115009, arXiv:0808.2512 [hep-ph].

[28] A. Chamblin, F. Cooper and G. C. Nayak, Phys. Lett. B672 (2009) 147.

[29] R. Emparan, G. T. Horowitz and R. C. Myers, Phys. Rev. Lett. 85, 499 (2000).

[30] J. Pumplin, et al., JHEP 0207 (2002) 102.

[31] M. M. Najafabadi and S. P. Mehdiabadi, arXiv:0803.1287 [hep-ph].

[32] N. Kidonakis, Phys. Rev. D 64, 014009 (2001); N. Kidonakis, E. Laenen, S. Moch and R. Vogt, Phys. Rev. D 64, 114001 (2001); R. Bonciani, S. Catani, M. L. Mangano and P. Nason, Nucl. Phys. B529, 424 (1998); W. Beenakker, W. L. van Neerven and J. Smith, Phys. Rev. D 40, 54 (1989); W. Beenakker, W. L. van Neerven, R. Meng, G. A. Schuler and J. Smith, Nucl. Phys. B351, 507 (1991); N. Kidonakis and R. Vogt, arXiv:0805.3844 [hep-ph]; E. Laenen, G. Oderdn and G. Sterman, Phys. Lett. B438, 173 (1998); P. Nason, S. Dawson and R. K. Ellis, Nucl. Phys. B 303, 607 (1988); M. Czakon and A. Mitov, arXiv:0811.4119; arXiv:0812.0353.

[33] N. Kidonakis and R. Vogt, Phys. Rev. D78 (2008) 074005, arXiv:0805.3844 [hep-ph].

[34] N. Kidonakis and R. Vogt, Phys. Rev. D68 (2003) 114014, hep-ph/0308222.

[35] B. S. Acharya, F. Cavallari, G. Corcella, R. D. Sipio, G. Petrucciani, arXiv:0806.0484 [hep-ex]; arXiv:0804.4122 [hep-ph]; W. Bernreuther, J.Phys.G35:083001,2008; T. Han, arXiv:0804.3178 [hep-ph]; M. Cacciari, S. Frixione, M. M. Mangano, P. Nason, G. Ridolfi, arXiv:0804.2800 [hep-ph]; T. Han, R. Mahbubani, D. G.E. Walker, L-T. Wang, arXiv:0803.3820 [hep-ph]; J.A. Aguilar-Saavedra, arXiv:0803.3810 [hep-ph]; U. Baur, L.H. Orr, Phys.Rev.D76:094012,2007; J. D'hondt, arXiv:0707.1247 [hep-ph]; N. Kidonakis, Phys.Rev.D75:071501,2007; V. Barger, T.Han, D. G.E. Walker, Phys.Rev.Lett.100:031801,2008; W. Wagner, Rept. Prog. Phys. 68, 2409 (2005); A. Quadt. E. Phys. J. C 48, 835 (2006); R. Kehoe, M. Narain and A. Kumar, Int. J. Mod. Phys. A 23, 353 (2008).

[36] L. A Anchordoqui, H. Goldberg, D. Lust, S. Nawata, S. Stieberger, T. R. Taylor, Phys.Rev.Lett.101:241803,2008, arXiv:0808.0497; arXiv:0904.3547v1 [hep-ph].

[37] R. Blumenhagen, B. Kors, D. Lust and S. Stieberger, Phys. Rept. 445, 1 (2007), hep-th/0610327. 
[38] D. Lust, S. Stieberger and T. R. Taylor, Nucl.Phys.B808:1-52,2009.

[39] L. F. Alday and J. Maldacena, L. F. Alday and J. M. Maldacena, JHEP 0706:064,2007, arXiv:0705.0303 [hep-th].

[40] J. McGreevy, A. Sever, JHEP 0802:015,2008, arXiv:0710.0393 [hep-th].

[41] A. Bhatti, et al., J. Phys. G36, 015004 (2009), arXiv:0807.4961 [hep-ph].

[42] A. Chamblin, F. Cooper and G. C. Nayak, Phys. Rev. D 69, 065010 (2004); F. Cooper, E. Mottola and G. C. Nayak, Phys. Lett. B 555, 181 (2003); G. C. Nayak et al., Nucl. Phys. A687, 457 (2001). 